CERN-TH 6923/93

BI-TP-93/32

\title{
TRANSITION FROM A QUARK-GLUON PLASMA IN THE PRESENCE OF A SHARP FRONT
}

\author{
N. Bilić1 ${ }^{1,2}$, J. Cleymans ${ }^{1}$, K. Redlich ${ }^{3,4}$ and E. Suhonen ${ }^{5,6}$
}

\begin{abstract}
The effect of a sharp front separating the quark-gluon plasma phase from the hadronic phase is investigated. Energy-momentum conservation and baryon number conservation constrain the possible temperature jump across the front. If one assumes that the temperature in the hadronic phase is $T \simeq 200 \mathrm{MeV}$, as has been suggested by numerous results from relativistic ion collisions, one can determine the corresponding temperature in the quark phase with the help of continuity equations across the front. The calculations reveal that the quark phase must be in a strongly supercooled state. The stability of this solution with respect to minor modifications is investigated. In particular the effect of an admixture of hadronic matter in the quark phase (e.g. in the form of bubbles) is considered in detail. In the absence of admixture the transition proceeds via a detonation transition and is accompanied by a substantial super-cooling of the quark-gluon plasma phase. The detonation is accompanied by less supercooling if a small fraction of bubbles is allowed. By increasing the fraction of bubbles the supercooling becomes weaker and eventually the transition proceeds via a smoother deflagration wave.
\end{abstract}

1 Department of Physics, University of Cape Town, Rondebosch 7700, South Africa

2 Rudjer Bošković Institute, P.O. Box 1016, Zagreb, Croatia

3 Fakultät für Physik, Universität Bielefeld, W-4800 Bielefeld, Germany

4 Department of Theoretical Physics, University of Wroclaw, PL-5025 Wroclaw, Poland

5 Department of Theoretical Physics, University of Oulu, SF-90550 Oulu, Finland

6 CERN, CH-1211 Geneva 23, Switzerland 


\section{Introduction}

Experimental results from heavy-ion collisions at CERN indicate that hadrons are being produced with a temperature of about $T \simeq 200 \mathrm{MeV}[1,2,3,4]$. From an analysis of the ratios of strange baryons as measured by the WA85 collaboration at CERN [2] one finds that a baryonic chemical potential of $\mu_{B}=300 \mathrm{MeV}$ is consistent with their results [5-12] (provided no transverse flow exists [13]). As a first attempt one can therefore assume that the thermodynamic properties of the hadronic gas are known. It is then of interest to investigate whether this hadronic gas could have been in a quarkgluon plasma phase before freeze-out. To this end we assume that a sharp front existed separating the two phases. Various conservation laws, e.g. baryon number, energy and momentum, restrict the temperature difference across the front. In addition, the second law of thermodynamics requires the entropy to increase across the front. It can easily be established that continuity in the various conservation laws leads to only one possibility, namely a highly supercooled quark-gluon plasma must have existed before the transition to the hadronic phase. The degree of supercooling is surprisingly high, $T_{q} \sim 70 \mathrm{MeV}$. This leads one to suspect whether some alternate mechanism started operating, preventing the quark-gluon plasma from supercooling to such a large degree. In this paper we would like to investigate several possibilities which could prevent this. In particular, it is known that supercooling enhances the formation of bubbles of hadronic matter. We will therefore consider that the quark phase was not pure but that some admixture of hadronic matter exists. It is known that the boiling of liquids only takes place if impurities are present or if some admixture of the gaseous state exists. Our results confirm this standard knowledge. The transition from quarkgluon plasma to hadrons proceeds in a violent manner towards superheated hadrons. The presence of an admixture of hadronic material in the form of bubbles or in any other form makes the transition easier. Our results agree with this expectation, from the moment the fraction of hadronic material is appreciable, the violent detonation type transition turns into a smoother deflagration type. The transition between the two solution shows an S-type of curve. This means that for a given reaction the solution is not unique. A possible interpretation would be that in this region a chaotic turbulent type of transition takes place.

The paper is organized as follows : in the next section we discuss the equations of state we use in the the quark-gluon plasma and in the hadronic phases; in section 3 we present for completeness the equations expressing energy, momentum and baryon

number conservation. We also discuss the adiabaticity condition; in section 4 we discuss the admixture of "impurities" into the quark phase in the form of hadronic matter. The velocities around the sharp front are discussed in section 5. In section 6 we present our conclusions and give a summary of our results.

\section{Equation of State in the Quark Gluon Plasma}

In this section we discuss the equation of state used in the quark- gluon plasma 
phase. We consider the cut-off model, motivated by numerical results from lattice gauge theories [14]. The precise value of the cut-off has a big influence on our results. For comparison we also consider the M.I.T. bag equation of state which has been previously discussed in detail [15]. In the cut-off model one leaves out the low momentum part of the particles, e.g. for gluons we have :

$$
n_{g}=16 \int_{k_{c}}^{\infty} \frac{d^{3} p}{(2 \pi)^{3}} \frac{1}{e^{E / T}-1}
$$

It is well-known that the cut-off model reproduces the lattice results reasonably well. It is not excluded that a better way to proceed is to give the gluon a temperature dependent mass as has been argued recently by Goloviznin and Satz [16]. This proposal cannot be followed up here because we want to include the possibility of super-cooling and heating and we, therefore, have to know the equation of state both below and above the critical temperature. We have chosen a value of $5 T_{c}$ for the cut-off parameter in order to reproduce the critical temperature $T_{c}$ obtained in lattice gauge theories for $\mu_{B}=0$ [17]. Certain quantities are very sensitive to the precise value of this cut-off and to show this influence we also present for comparison results obtained with a value of $3.5 T_{c}$. The corresponding results for the energy density are shown in Fig. 1. On the hadronic side we incorporate the hard core radius of hadrons by choosing [18]

$$
\epsilon_{h}=\frac{\epsilon_{h}^{0}}{1+V_{0} n_{h}^{0}},
$$

where the subscript zero refers to point-like quantities, $V_{0}$ refers to a typical proper volume of a hadron which we take to have a radius given by $0.8 \mathrm{fm}$. The hadronic side in our approach is a composition of non-interacting quantum gases. The use of quantum statistics is essential since in a wide range of $\mu_{B}$ and $T$ the Boltzmann approximation is not satisfied. We include all well established hadronic resonances listed in the latest edition of the Review of Particle Properties [19]. A correction similar to equation (11) is performed to all thermodynamic quantities. The behaviour suggested by the M.I.T.-bag equation of state is very different and is not supported by the lattice results. The larger cut-off reduces the energy-density in the quark-gluon plasma since a big contribution is being left out. The latent heat thus becomes very small for a large cut-off. Since lattice results are only available for zero chemical potential, $\mu_{B}=0$, it is not immediately clear how to generalize the cut-off model to finite baryon densities. Assuming the cut-off to be independent of $\mu_{B}$ we have determined the phase diagram depicted in Fig. 2 where we compare it with the M.I.T.-bag model one. At $\mu_{B}=0$ the two are chosen to coincide. For non-zero values of $\mu_{B}$ they become more and more different. For the region we are interested in $\mu_{B} \sim 300 \mathrm{MeV}$ and the difference is not very large. Having discussed the equations of state we will be using in each phase, we now proceed to the equations of continuity across the sharp front. 


\section{Equations of Continuity across the Boundary}

Energy and momentum conservation across the front leads to the well-known two equations (see e.g. [20]) :

$$
\begin{aligned}
\left(\epsilon_{h}+P_{h}\right) \gamma_{h}^{2} v_{h}+P_{h} & =\left(\epsilon_{q}+P_{q}\right) \gamma_{q}^{2} v_{q}+P_{q}, \\
\left(\epsilon_{h}+P_{h}\right) v_{h}^{2} \gamma_{h}^{2} & =\left(\epsilon_{q}+P_{q}\right) v_{q}^{2} \gamma_{q}^{2},
\end{aligned}
$$

both sides of the front are at different temperatures and chemical potentials. An important remark here : $\epsilon_{q}$ refers to the quark-gluon plasma side of the sharp front, where we allow for an admixture of hadronic matter consisting for simplicity of only pions. Thus

$$
\epsilon_{q}=(1-f) \epsilon_{q}^{0}+f \epsilon_{\pi}
$$

where $\epsilon_{q}^{0}$ refers to the pure quark-gluon matter and $f$ is the fraction of hadronic matter present in the quark-gluon plasma phase. We will consider $f$ to be a free parameter and investigate how various quantities depend on it. It turns out that for the small cut-off as well as for the bag model the transition temperature and chemical potential are not sensitive to variation of $f$ provided that it is not very large. Fore the large cut-off the transition changes completely its nature when $f$ becomes of the order of $20 \%$. The thermodynamic quantities (pressure, energy density) refer to the rest frame quantities. The index $q$ refers to the quark-gluon plasma side which has an admixture of hadronic matter, while the index $h$ refers to the hadronic phase, $v$ refers to the velocity of the gas in each phase with respect to the front, $\gamma$ is the standard relativistic factor $\left(1 / \sqrt{1-v^{2}}\right)$. In addition to the above two equations, baryon number conservation across the front leads to

$$
n_{h} v_{h} \gamma_{h}=n_{q} v_{q} \gamma_{q}
$$

where $n_{h}$ and $n_{q}$ refer to the baryon densities on the hadronic and on the quark-gluon plasma sides respectively.

In order to determine the four unknown quantities $\epsilon_{q}, P_{q}, v_{q}$ and $v_{h}$ as functions of $\epsilon_{h}, P_{h}$ and $n_{h}$ we need one more equation. This is given by the requirement of non-decreasing entropy which implies in our notation

$$
s_{h} v_{h} \gamma_{h} \geq s_{q} v_{q} \gamma_{q} \quad,
$$

or, combining (6) and (7)

$$
\frac{s_{h}}{n_{h}} \geq \frac{s_{q}}{n_{q}} .
$$

As has been shown previously [15], eqs. $(3,4,6,7)$ yield a supercooled quark-gluon plasma. The equality sign in (8), corresponding to an adiabatic process, determines the minimal amount of supercooling needed to fit the hadronic data without violating the second law of thermodynamics. Therefore, we may use the equation

$$
\frac{s_{h}}{n_{h}}=\frac{s_{q}}{n_{q}},
$$


as the fourth constraint, keeping in mind that the quark-gluon temperature $T_{q}$ thus obtained is maximal.

Equations $(3,4,6)$ can be solved for the velocities, leading to

$$
\begin{gathered}
v_{q}^{2}=\frac{\left(P_{h}-P_{q}\right)\left(\epsilon_{h}+P_{q}\right)}{\left(\epsilon_{h}-\epsilon_{q}\right)\left(\epsilon_{q}+P_{h}\right)}, \\
v_{h}^{2}=\frac{\left(P_{h}-P_{q}\right)\left(\epsilon_{q}+P_{h}\right)}{\left(\epsilon_{h}-\epsilon_{q}\right)\left(\epsilon_{h}+P_{q}\right)} .
\end{gathered}
$$

With help of eq. (6) the velocities can be eliminated yielding

$$
\left(\frac{n_{q}}{n_{h}}\right)^{2}=\frac{\left(\epsilon_{q}+P_{q}\right)\left(\epsilon_{q}+P_{h}\right)}{\left(\epsilon_{h}+P_{h}\right)\left(\epsilon_{h}+P_{q}\right)}
$$

Combining now equations (9) and (12) we can calculate numerically the temperature $T_{q}$ and the chemical potential $\mu_{B}^{q}$. This in turn yields all thermodynamic quantities on the quark-gluon side. In the following sections we discuss our main results.

\section{Admixture of Hadronic Matter}

In Fig. 3 we show our results for the case when there is no admixture of hadronic matter in the quark-gluon plasma phase. There is a substantial amount of supercooling in the quark phase. The transition then takes place to a hadronic gas which has a temperature $T=200 \mathrm{MeV}$ as suggested by most experiments at CERN using a relativistic ion beam. Most results from lattice gauge theories indicate that this temperature is higher than the critical temperature of the phase transition. We thus have to conclude that the hadronic gas is in a super-heated phase.

This scenario immediately raises the following question : what happens if other mechanisms prevent this strong super-cooling? In particular it has been argued convincingly by Csernai and Kapusta [21] that super-cooling enhances the formation of hadronic bubbles in the quark-gluon plasma phase. We have therefore considered admixing hadronic matter to the quark-gluon plasma phase. We expected that the strong degree of supercooling would disappear however, we did not expect our results to be so sensitive on the value of the cut-off chosen for the equation of state in the quark-gluon plasma phase. This is shown in Fig. 4. In this figure we keep the parameters on the hadronic side fixed at $T=200 \mathrm{MeV}$ and $\mu_{B}=300 \mathrm{MeV}$ but we vary the amount of hadronic admixture, given by the fraction $f$ as introduced in equation (4), on the quark side and we calculate each time the resulting value of the temperature on the other side of the sharp front. For simplicity the admixture is in the form of pions only. If the cut-off value is large, $5 T_{c}$, then there is an abrupt turnover when the admixture exceeds $20 \%$. The temperature increase across the front is reduced after this. However when the cut-off is taken at $3.4 T_{c}$ then the picture is completely different : the strong supercooling persists anomalously long until the admixture has reached more than $60 \%$, 
in which case it is no longer possible to speak of a "small" admixture. If a bag-model equation of state is used the behavior is very similar to the case of the small cut-off.

In Fig. 5 we show the baryon chemical potential $\mu_{q}$ on the quark-gluon plasma side. The observed behaviour follows the pattern already found for the temperature in Fig. 4. As previously, the cut-off in the equation of state is very important.

\section{Velocities}

It is customary to work in the frame where the sharp front separating the two phases is at rest. In this frame the quark-gluon plasma moves towards the front with a velocity $v_{q}$ and the hadrons move away from the front with a velocity $v_{h}$. If the two velocities are different, as is usually the case, one observes a phenomenon of pile up on one side, e.g. if the quarks have the largest velocity, the hadrons will pile up with a large baryon density at the other side of the front. If $v_{q}<v_{h}$ one has a deflagration, if $v_{q}>v_{h}$ one has a detonation (see [22-24]).

The velocities can be easily calculated from equations (10) and (11). Our results for $v_{q}^{2}$ and $v_{h}^{2}$ as functions of the fraction $f$ of hadronic matter mixed into the quark-gluon phase are shown in Figs. 6 and 7 respectively. In Fig. 8 we plot the ratio of $v_{h}$ to $v_{q}$ as a function of $f$ and in Fig. 9 the relative velocity defined as

$$
v_{r e l}=\frac{v_{h}-v_{q}}{1-v_{h} v_{q}}
$$

The change in the transition mechanism leads to very sharp variations in $v_{q}$ and $v_{h}$. The velocities become unphysical along the curves between the points A and A'. The detonation and deflagration branches are clearly separated in Figs. 6 and 7 whereas in Figs. 8 and 9 the detonation takes place below the point $\mathrm{A}$ and deflagration above the point A'.

As is well known, small perturbations in a medium propagate with the velocity of sound in that medium. It is therefore of special interest to compare $v_{q}$ and $v_{h}$ with the velocity of sound in each phase. The latter is defined as [20]

$$
c_{s}^{2}=\left.\frac{\partial P}{\partial \epsilon}\right|_{s / n}
$$

To calculate the speed of sound we write eq. (14) as

$$
c_{s}^{2}=\frac{\partial P / \partial T+\partial P / \partial \mu_{B} \cdot d \mu_{B} / d T}{\partial \epsilon / \partial T+\partial \epsilon / \partial \mu_{B} \cdot d \mu_{B} / d T},
$$

where the derivative $d \mu_{B} / d T$ is taken such that the ratio $s_{h} / n_{h}$ remains constant. Thus

$$
\frac{d \mu_{B}}{d T}=-\frac{n \partial s / \partial T-s \partial n / \partial T}{n \partial s / \partial \mu_{B}-s \partial n / \partial \mu_{B}}
$$


The velocities of sound in the quark-gluon and in the hadronic phases are shown in Figs. 6 and 7 as functions of $f$.

The points O, O', A and A' divide the curves in Figs. 6-9 according to the magnitudes of the velocities of sound in the corresponding media. The following inequalities hold :

below $\mathrm{O}: v_{q}>c_{s, q} \quad, \quad v_{h}<c_{s, h}$

on $\mathrm{OA}: v_{q}<c_{s, q}, \quad v_{h}<c_{s, h}$

on $\mathrm{A}^{\prime} \mathrm{O}^{\prime}: v_{q}>c_{s, q} \quad, \quad v_{h}>c_{s, h}$

above $\mathrm{O}^{\prime}: v_{q}<c_{s, q} \quad, \quad v_{h}>c_{s, h}$

The comparison of $v_{q}$ and $v_{h}$ with the velocities of sound in the corresponding media shows that the conditions for the stability of the front

$$
v_{q}>c_{s, q} \quad ; \quad v_{h}<c_{s, h}
$$

are fulfilled only along the upper part of the detonation branch. Following the arguments presented in Ref. [20] one would conclude that the rest of the curve is unstable and the front would disintegrate. Condition (17) is however derived under the assumption of a non-decreasing entropy density, i.e. for $s_{h} \geq s_{q}$. In the present situation we require only the entropy per baryon not to decrease which means that $s_{h}<s_{q}$ is physically acceptable provided the condition expressed in eq. (8) is satisfied. Indeed we find that the ratio $s_{q} / s_{h}$ becomes less than one along the detonation branch (corresponding to the part below A' on Figs. 8 and 9) and $s_{q} / s_{h}>1$ along the deflagration branch (above $\left.A^{\prime}\right)$.

\section{Conclusions}

In this paper we have investigated the consequences arising from the existence of a sharp front separating the quark-gluon plasma phase from the hadronic phase. The equations of continuity for energy, momentum and baryon number as well as the constraint following from the second law of thermodynamics (entropy increase), require a substantial amount of supercooling to occur in the quark-gluon plasma before the transition can take place. This transition then leads to a highly super-heated hadronic gas. We have also investigated what happens if the quark- gluon plasma is not pure but contains an admixture of hadronic matter. It is known that supercooling is limited when "impurities" are present in the super-cooled phase. We found that from the moment when the admixture of hadronic matter is more than about $20 \%$ of the total (the fraction refers to the energy content, not to the particle content) then the transition does not take place via a detonation type of wave but via a deflagration one. In this case the transition is smoother and the temperature jump across the sharp front is not as pronounced as in the absence of admixture of hadronic matter.

The crossover region between the two solution shows a remarkable S-shape structure. The multiple valued $T_{q}$ and $\mu_{B}^{q}$ in that region indicate that in the given range of $f$ a chaotic turbulent type of transition could take place. 
In summary, we have considered a model based on the existence of a sharp front separating the hadronic from the quark-gluon phase. We have arrived at the conclusion that in this situation the transition can only proceed if a substantial amount of supercooling takes place in the quark-gluon plasma phase if the transition is between pure phases. An admixture of hadronic material in the quark-gluon plasma phase makes the transition proceed in a much smoother way.

Acknowledgment We acknowledge continuing stimulating discussions in this field with H. Satz. Two of us (E.S. and K.R.) thank the Physics Department of the University of Cape Town for its hospitality. N.B. and J.C. would like to thank the hospitality of the Fakultät für Physik of the University of Bielefeld where this work was completed. (KR) also acknowledges the financial support by the German Federal Ministry for Science and Technology (BMFT). 


\section{References}

1) J. Bartke et al. (NA35), Z. Phys. C48 (1990) 191;

R. Stock et al. (NA35), Nucl. Phys. A525 (1991) 221c;

2) S. Abatzis et al. (WA85), Phys. Lett. B244 (1990) 130;

S. Abatzis et al. (WA85), Phys. Lett. B259 (1991) 508.

3) E. Andersen et al., Phys. Lett. B294 (1992) 127.

4) A. Franz (NA44), Report at the XXVI International Conference on High Energy Physics, 6.-12.8.1992, Dallas/Texas, USA.

5) J. Cleymans and H. Satz, Z. Phys., C57 (91993) 135.

6) J. Cleymans, K. Redlich, H. Satz and E. Suhonen, Z. Phys., C58 (1993) 347.

7) J. Rafelski, Phys. Lett. B262 (1991) 333.

8) J. Rafelski, Nucl. Phys. A544 (1992) 279c.

9) J. Letessier, A. Tounsi, U. Heinz, J. Sollfrank and J. Rafelski, Paris Preprint PAR/LPTHE/92-37, September 1992.

10) N.J. Davidson, H.G. Miller, R.M. Quick and J. Cleymans, Phys. Lett. B255 (1991) 195.

11) D.W. von Oertzen, N.J. Davidson, R.A. Ritchie and H.G. Miller, Phys. Lett. B274 (1992) 128.

12) N.J. Davidson, H.G. Miller, D.W. von Oertzen and K. Redlich, Z. Phys. C56 (1992) 319.

13) E. Schnedermann and U. Heinz, Phys. Rev. Lett. 69 (1992) 2908.

14) F. Karsch, Z. Phys. C38 (1988) 147;

M. Gorenstein and O. Mogilevsky, Z. Phys. C38 (1988) 161;

J. Engels, J. Fingberg, K. Redlich, H. Satz and M. Weber,

Z. Phys. C42 (1989) 341;

J. Engels, J. Fingberg, F. Karsch, D. Miller and M. Weber,

Phys. Lett. B252 (1990) 625.

15) N. Bilić, J. Cleymans, E. Suhonen and D.W. von Oertzen, Cape Town preprint UCT-TP 93/191, Phys. Lett. B (1993) (in press).

16) V.V. Goloviznin and H. Satz, CERN preprint CERN-TH /93.

17) S. Gottlieb et al., Phys. Rev. Lett. 59 (1987) 1881.

18) J. Cleymans, K. Redlich, H. Satz and E. Suhonen, Z. Phys. C33 (1986) 151.

19) Particle Data Group, Phys. Rev. D45 (1992) S1.

20) L. Landau and E.M. Lifshitz, Hydrodynamics, Pergamon Press, London (1959).

21) L. Csernai and J. Kapusta, Phys. Rev. Lett. 67 (1993) 737.

22) L. Van Hove, Z. Phys. C21 (1983) 93; C27 (1985) 135. 
23) M. Gyulassy, K. Kajantie, H. Kurki-Suonio and L. McLerran, Nucl. Phys. B237 (1984) 477.

25) K.S. Thorne, Astrophys. J. 179 (1973) 897. 


\section{Figure Captions:}

1: Energy-density as a function of temperature at fixed $\mu_{B}=0.30 .3 \mathrm{GeV}$. The longdashed line corresponds to the equation of state of the M.I.T.-bag with bag constant $\mathrm{B}=0.2 \mathrm{GeV} / \mathrm{fm}^{3}$, the dashed line is the cut-off model with a value $3.4 T_{c}$ for the cutoff while for the long dash-dotted line the cut-off is $5 T_{c}$. The solid line corresponds to the energy-density as calculated from the hadronic gas. The critical temperature is indicated by the dotted line at $T_{c}=150 \mathrm{MeV}$.

2: The phase diagram calculated in the cut-off model and in the M.I.T. bag model. At $\mu_{B}=0$ the bag constant is chosen such as to reproduce the same critical temperature as the cut-off model. The value of the cut-off is taken to be $5 T_{c}$ independent of $\mu_{B}$.

3: The energy-density of the hadronic gas (lower curve) and of the quark-gluon plasma (upper curve) as a function of temperature. Super-cooling and super-heating are indicated by the dashed line. The transition from the super-cooled quark-gluon plasma phase to the hadronic gas phase is indicated by the long-dashed line.

4: The quark temperature $T_{q}$ versus the fraction of hadronic admixture in the quarkgluon plasma phase. The solid line corresponds to a cut-off value of $5 T_{c}$, the dashed line to $3.4 T_{c}$. The dash-dotted line is for the equation of state of the M.I.T.-bag model.

5: The baryon chemical potential in the quark-gluon plasma phase versus the fraction of hadronic admixture in the quark-gluon plasma phase. The solid line corresponds to a cut-off value of $5 T_{c}$, the dashed line to $3.4 T_{c}$. The dash-dotted line is for the equation of state of the M.I.T.-bag model.

6: The velocity squared of the quark matter (full line). The value of the cut-off parameter in the equation of state is $5 T_{c}, f$ is the admixture fraction. The dashed line is the velocity of sound squared in quark matter. See text for further explanations.

7: The velocity squared of the hadronic gas (full line). The value of the cut-off parameter in the equation of state is $5 T_{c}, f$ is the admixture fraction. The dashed line is the velocity of sound in the hadronic matter. See text for further explanations.

8: Ratio of the hadronic gas velocity over the quark phase velocity as a function of the admixture fraction $\mathrm{f}$. The value of the cut-off parameter in the equation of state is $5 T_{c}$. If the ratio is less than one, the transition proceeds via a detonation wave, if it is above one, it proceeds via a deflagration wave. Between the points A and A' the velocities are unphysical. See text for further explanations.

9: The difference between the two velocities. The value of the cut-off parameter in the equation of state is $5 T_{c}, f$ is the admixture fraction. Between the points $\mathrm{A}$ and A' the velocities are unphysical See text for further explanations. 